\section{Tendência secular da peste no Estado do Ceará, Brasil}

\author{
Secular plague trend in Ceará State, Brazil
}

\begin{abstract}
After its introduction into the State of Ceará, Brazil, in 1900, the plague was established in seven ecological complexes: Chapada do Araripe and the Ibiapaba, Baturité, Machado, Matas, Pedra Branca, and Uruburetama mountains. These natural foci were monitored successively from 1935 to 2004 by the National Health Department, National Plague Service, National Department of Rural Endemics, Superintendency of Public Health Campaigns, National Health Foundation, and finally by the National Health Surveillance Secretariat. Data analysis on human cases during these 70 years allowing identifying different plague circulation patterns in the human population, alternating high incidence with silent periods and characterizing a chronological periodicity with unique epidemiological characteristics, besides concluding that plague should still be considered a potential threat, thus justifying the revitalization of surveillance measures by strengthening all levels in the Unified National Health System.
\end{abstract}

Plague; Yersinia pestis; Epidemiologic Surveillance; Zoonoses

\author{
Antonia Ivoneida Aragão 1 \\ Ricardo José Soares Pontes 2 \\ Antônio Carlos Mendonça Seoane 3 \\ Osmar José do Nascimento 1 \\ Celso Tavares 4 \\ Alzira Maria Paiva de Almeida 5
}

\section{Introdução}

A peste, infecção pela Yersinia pestis, é uma zoonose de roedores silvestres transmitida usualmente pela picada de pulgas e constitui um risco potencial para o homem e outros animais que freqüentem os ecossistemas da infecção, os focos, distribuídos por todos os continentes, exceto a Oceania 1,2,3. No ser humano, a doença se expressa habitualmente por três formas clínicas: ganglionar, septicêmica e pulmonar ${ }^{2}$. É um problema de saúde pública de grande importância epidemiológica por seu potencial epidêmico e elevada letalidade, o que a inclui na classe I do Regulamento Sanitário Internacional vigente, sendo atualmente considerada uma doença reemergente e potencial arma bacteriológica 1,4,5, o que exige a revitalização das ações específicas de vigilância epidemiológica.

A zoonose foi introduzida no Brasil pelo porto de Santos, Estado de São Paulo, na vigência da terceira pandemia, em outubro de 1899, e a partir de então se disseminou e afligiu várias cidades do litoral, atingindo Fortaleza, Estado do Ceará, em 1900. O Governo Federal instituiu, então, campanhas profiláticas que incluíam o combate aos hospedeiros e vetores, bem como o tratamento e isolamento dos doentes. Essas campanhas conseguiram erradicar a peste dos grandes centros urbanos, mas não impediram a sua propagação. A infecção irradiou-se para o interior do país a partir de 1906, através das vias férreas e estradas, 
estabelecendo focos dispersos pelo semi-árido do Ceará, Rio Grande do Norte, Paraíba, Pernambuco, Alagoas, Bahia e de Minas Gerais e um isolado, no Rio de Janeiro 6,7.

As atividades de controle desenvolvidas pelos estados a partir de 1936 passaram para o âmbito do Departamento Nacional de Saúde (DNS), ganhando consistência científica e unidade. Após 1941, com a criação do Serviço Nacional de Peste (SNP), elas se tornaram mais eficazes, possibilitando refrear o avanço da peste, uma vez que se passou a investir na epidemiologia e na qualidade do pessoal envolvido nas ações. Em 1956, o SNP foi incorporado ao Departamento Nacional de Endemias Rurais (DNERu) e em 1970 foi criada a Superintendência de Campanhas de Saúde Pública (SUCAM), que estruturou uma rede de laboratórios no país cobrindo os focos com atividade. Em 1990, a SUCAM foi extinta e criada a Fundação Nacional de Saúde (FUNASA).

No decorrer da década de 90, com a progressiva implantação do Sistema Único de Saúde (SUS) no país, instituindo como princípio básico a descentralização político-administrativa, enfatizou-se a municipalização dos serviços, o que favoreceria o desenvolvimento das ações de vigilância epidemiológica. No Ceará, a descentralização das ações de controle das endemias ocorreu a partir de 2001, com a Secretaria Estadual de Saúde (SESA/CE) assumindo as históricas e tradicionais atribuições da FUNASA e adequandoas à estrutura do estado, com as áreas pestígenas sendo distribuídas por 12 Células Regionais de Saúde (CERES). Em 2003, o Programa de Controle da Peste (PCP) passou à responsabilidade da Secretaria de Vigilância em Saúde do Ministério da Saúde (SVS/MS).

O objetivo deste trabalho foi analisar o comportamento epidemiológico e as estratégias de vigilância da peste nos diversos focos cearenses no período de 1935 a 2004, em contextos tão distintos como o da centralização absoluta que caracterizou o DNS e o SNP e o da descentralização vigente, com o desenvolvimento de ações de vigilância e controle regionalizadas e desenvolvidas pelas CERES.

\section{Materiais e métodos}

O levantamento de dados sobre os casos notificados de peste humana, tanto os positivos Classe 1 (confirmados laboratorialmente) quanto os Classe 2 (confirmados por evidência clínicoepidemiológica) 8 , foi realizado nos arquivos da FUNASA e da SESA/CE. Os registros da FUNASA contêm informações geradas a partir de 1935 pelo DNS, SNP, DNERu e SUCAM até o ano 2000, quando as ações de vigilância foram descentralizadas para os Estados. Os dados da SESA/CE cobrem o período de 2001 a 2004.

\section{Resultados}

\section{Foco da Chapada do Araripe} (CERES 19, 20 e 21)

O Complexo Ecológico da Chapada do Araripe estende-se pelo oeste de Pernambuco, sul do Ceará e leste do Piauí, e os 11 municípios cearenses que compõem o foco estão distribuídos administrativamente por três CERES: a 19 (três municípios); a 20 (cinco) e a 21 (três) (Figura 1, Tabela 1).

Os dados analisados (Figura 2) mostram que em 1935 o foco achava-se em plena atividade e em 1936, de fevereiro a junho, foram notificados 93 casos no Crato. Observou-se um declínio em 1937, com retorno à atividade no período de 1944 a 1950 e, após dez anos de silêncio, novos casos ocorreram entre 1961 e 1969. Em 1973, iniciando-se em Santana do Cariri, a região foi varrida por novo surto. Os últimos casos humanos confirmados aconteceram em 1976, em Crato e Jardim.

\section{Foco da Serra da Ibiapaba} (CERES 11, 13 e 15)

O Complexo Ecológico da Serra de Ibiapaba é composto por 13 municípios, e os 12 com atividade pestosa estão distribuídos por três diferentes CERES: a 11 (dois municípios), a 13 (oito) e a 15 (dois) (Figura 1, Tabela 1). Esta última, com sede em Crateús, estende-se até o foco da serra das Matas, que também faz parte da CERES 5, sediada em Canindé. É um dos focos mais importantes do Ceará, donde atualmente provém a maioria das notificações de casos suspeitos. Os dados analisados mostram intensa atividade de 1935 a 1951 (Figura 2) e após três anos de silêncio ocorreu um caso isolado em Ipu e três em Ipueiras.

Em 1966, dando início a um período de intensa atividade, eclodiram novos casos em Ipu e Ipueiras que inicialmente foram descritos como "adenites febris de etiologia desconhecida", cuja elucidação ocorreu após rigorosa investigação desenvolvida no período de 1971/1972. A Y. pestis foi isolada de material obtido por digitotomia em cadáver de um paciente residente em Guaraciaba do Norte, e de aspirado de bubão e/ou sangue de 16 pacientes (nove de Guaraciaba do Norte, cinco de Ipu, um de Ipueiras e um de São Benedito). Constatou-se um declínio em 1986 e, novamente, 


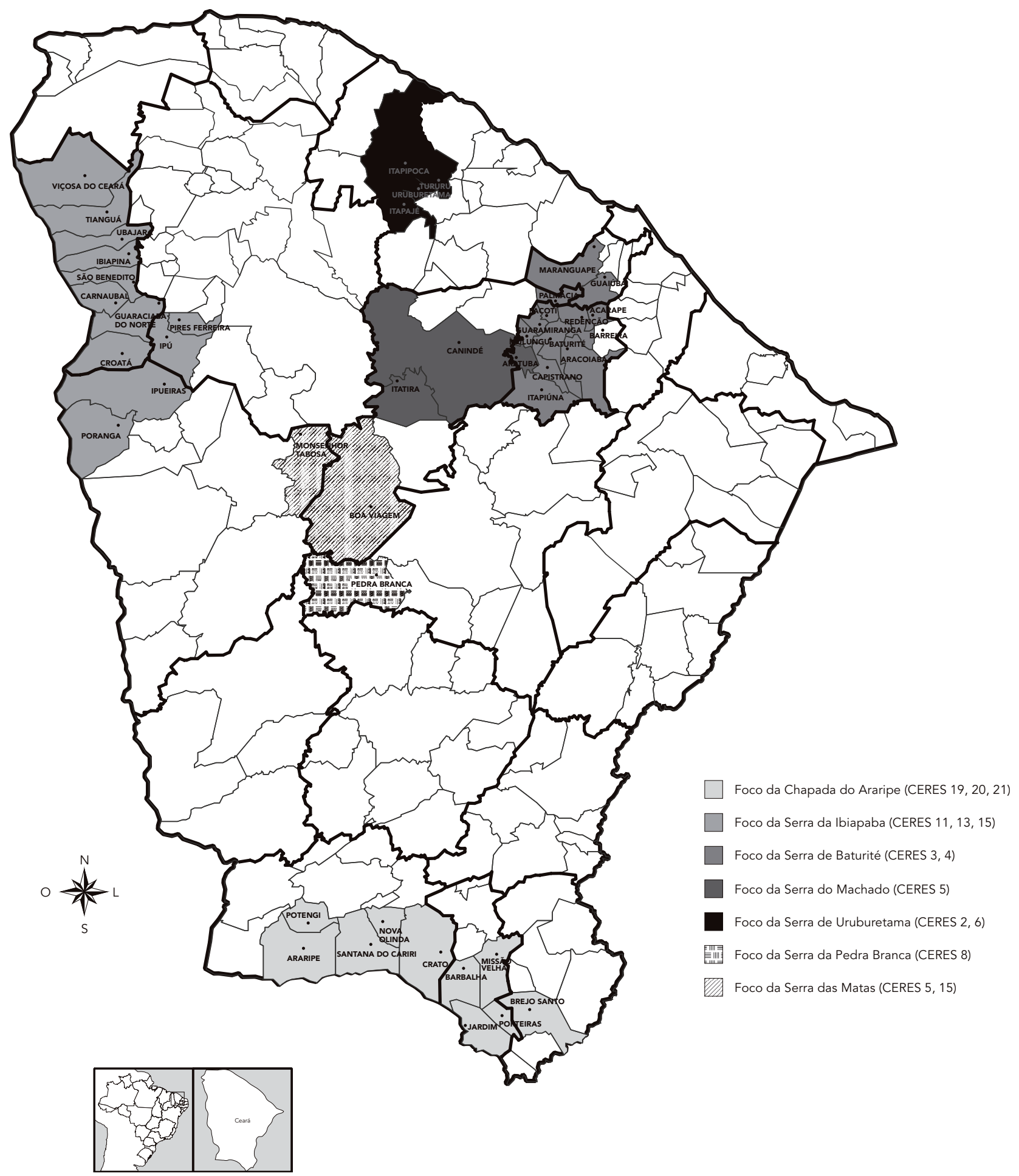


Distribuição dos municípios pestígenos pelas áreas de foco e pelas Células Regionais de Saúde (CERES), Ceará, Brasil.

\begin{tabular}{|c|c|c|}
\hline Focos & CERES & Municípios \\
\hline \multirow[t]{3}{*}{ Chapada do Araripe } & 19. Brejo Santo & Abaiara, Brejo Santo, Porteiras \\
\hline & 20. Crato & Araripe, Crato, Nova Olinda, Potengi, Santana do Cariri \\
\hline & 21. Juazeiro do Norte & Barbalha, Jardim, Missão Velha \\
\hline \multirow[t]{4}{*}{ Serra da Ibiapaba } & 11. Sobral & Ipu, Pires Ferreira \\
\hline & 13. Tianguá & Carnaubal, Croata, Guaraciaba do Norte, Ibiapina, São Benedito, \\
\hline & & Tianguá, Ubajara, Viçosa do Ceará \\
\hline & 15. Crateús & Ipueiras, Poranga \\
\hline \multirow[t]{3}{*}{ Serra de Baturité } & 3. Maracanaú & Acarape, Maranguape, Palmácia, Guaiuba, Redenção \\
\hline & 4. Baturité & Aracoiaba, Aratuba, Baturité, Capistrano, Guaramiranga, Itapiúna, \\
\hline & & Mulungu, Pacoti \\
\hline Serra do Machado & 5. Canindé & Canindé, Itatira \\
\hline \multirow[t]{2}{*}{ Serra de Uruburetama } & 2. Caucaia & Itapajé \\
\hline & 6. Itapipoca & Itapipoca, Uruburetama \\
\hline Serra da Pedra Branca & 8. Quixadá & Pedra Branca \\
\hline \multirow[t]{2}{*}{ Serra das Matas } & 5. Canindé & Boa Viagem \\
\hline & 15. Crateús & Monsenhor Tabosa \\
\hline
\end{tabular}

um incremento da infecção em 1994 e 1996 em Guaraciaba do Norte, e em 1997 em Ipu.

\section{Foco da Serra de Baturité (CERES 3 e 4)}

O Complexo Ecológico da Serra de Baturité possui 13 municípios distribuídos por duas CERES: a 3 (cinco municípios) e a 4 (oito) (Figura 1, Tabela 1). Distando apenas $100 \mathrm{~km}$ de Fortaleza, a capital do Estado, é um foco que exige a atenção das equipes municipais de vigilância, pois sempre há o risco de transferência de casos de peste indevidamente diagnosticados e suas possíveis conseqüências, a pneumonia pestosa, por exemplo, bem como o transporte passivo de pulgas infectadas. Há registros de casos em Baturité e Pacoti em 1935 e 1936, e após cinco anos de silêncio a peste voltou a grassar novamente até 1948 . Em 1951 foram registrados mais quatro casos e depois de dez anos houve um surto em Palmácia, que se estendeu até Baturité, Aratuba, Capistrano, Itapiúna, Itatira (Serra do Machado), Boa Viagem (Serra das Matas) e Pedra Branca (Serra da Pedra Branca) (Figura 2).

Durante a década de 70 ocorreram alguns surtos e em 1978 foram registrados cinco casos de peste bubônica confirmados bacteriologicamente. Em Aratuba, um deles evoluiu para a for- ma pneumônica, o que gerou uma situação de altíssimo risco. Na década de 80 irrompeu um surto que atingiu vários municípios e em 1982, nos meses de agosto/novembro, foram notificados 36 casos humanos positivados sorologicamente, e os últimos casos registrados datam de 1985 (Figura 2).

\section{Foco da Serra do Machado (CERES 5)}

O Complexo Ecológico da Serra do Machado é composto por Canindé e Itatira, ambos localizados na Ceres 5, e a área pestígena abrange os dois municípios (Figura 1, Tabela 1). O primeiro só foi atingido em 1968 e o segundo a partir de 1962 por sucessivas ocorrências até 1971, quando aconteceu o último caso humano, numa extensão do surto iniciado em Palmácia (Figura 2). Deve-se assinalar que a CERES 5 também responde pelo Município de Boa Viagem, que compõe o foco da Serra das Matas.

\section{Foco da Serra de Uruburetama (CERES 2 e 6)}

O Complexo Ecológico da Serra de Uruburetama é constituído por quatro municípios e a área pestígena atinge três municípios distribuídos por duas CERES: a 2 (um município) e a 6 (dois) 
Figura 2

Distribuição dos casos humanos de peste pelas regiões pestígenas do Estado do Ceará, Brasil, 1935 a 2004.

2a) $1935-1969$

\begin{tabular}{|c|c|c|c|c|c|c|c|c|c|c|c|c|c|c|c|c|c|c|c|c|c|c|c|c|c|c|c|c|c|c|c|c|c|c|c|}
\hline Município & 35 & \begin{tabular}{|l|}
36 \\
\end{tabular} & 37 & 38 & 39 & 40 & 41 & 42 & 43 & 44 & 45 & 46 & 47 & 48 & & 50 & 51 & 52 & \begin{tabular}{|l|l|}
2 & 53 \\
\end{tabular} & \begin{tabular}{|l|l|} 
& 54 \\
\end{tabular} & \begin{tabular}{|l|l}
4 & 55 \\
\end{tabular} & & 57 & 58 & 59 & 60 & 61 & 62 & 63 & 64 & 65 & 66 & \begin{tabular}{|l|}
67 \\
\end{tabular} & & 69 \\
\hline Abaiara & & & & & & & & & & & & & & & & & & & & & & & T & & & & & 1 & & & $T$ & $T$ & & & \\
\hline Araripe & & & & & & & & & & & & & & & & & & & ] & 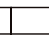 & 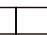 & & & & & & & 1 & 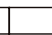 & & 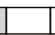 & t & & T & \\
\hline Barbalha & & & & I & & & & & & & & & & & & & & & & & & & & & & & & & & & & & & & \\
\hline Brejo Santo & & & 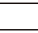 & & & & & & & & & & & & & & & & & & & & & & & & & 1 & d & & & 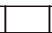 & & E & \\
\hline Crato & & & & & & & & & & & & & & & & & & & & & & & & & & & & 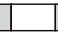 & & & & & & & \\
\hline Jardim & & & & & & & & & & & & & & & & & & & & & & & & & & & & T & & & & & & & \\
\hline Missão Velha & & & & & & & & & & & & & & & & & & & & & & & & & & & 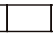 & I & & & & & & 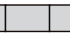 & \\
\hline Nova Olinda & & & & & & & & & & & & & & & & & & & & & & & & & & & 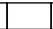 & 1 & & & & 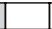 & & 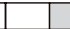 & \\
\hline Porteiras & & & & & & & & & & & & & & & & & & & & & & & & & & & & t & & & & & & 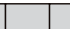 & \\
\hline Santana do Cariri & & & & & & & & & & & & & & & & & & & & & & & & & & & & 1 & & & & & & | & \\
\hline Guaraciaba do Norte & & & & & & & & & & & & & & & & & & & & & & & & & & & & T & & & & & & t & \\
\hline Ipu & & & & & & & & & & & & & & & & & & & & & & & & & & & & 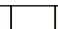 & & & & 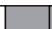 & | & 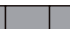 & \\
\hline Ipueiras & & & & & & & & & & & & & & & & & & & & & & & & & & & & 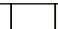 & & & & t & D & 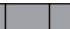 & \\
\hline Ibiapina & & & & I & & & & & & & & & & & & & & & & & & & & & & 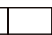 & & 1 & & & & L & & I & \\
\hline Poranga & & & & I & I & & & & & & & & & & & & & & & & & & & & & ] & & L & & & & 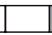 & & E & \\
\hline São Benedito & & & & & & & & & & & I & I & & & & & & & & & & & & & & & & 1 & & & & L & & 1 & \\
\hline Tianguá & & & & & & & & & & & I & & & & & & & & & 1 & & & & & & 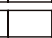 & T & 1 & & & & E & & E & \\
\hline Ubajara & & & & I & 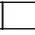 & & & & & & 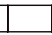 & I & & & & & & & & & & & & & & & 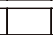 & I & & & & E & & 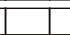 & \\
\hline Viçosa do Ceará & & & & & & & & & & & & & & & & & & & & & & & & & & & & & & & & & & & \\
\hline Aratuba & & & & & & & & & & & & & & & & & & & & & & & & & & & & . & & & & 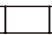 & & 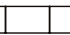 & \\
\hline Baturité & & & & & & & & & & & & & & & & & & & & & & & & & & & & & & & & & & 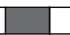 & \\
\hline Capistrano & & & & & 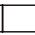 & & & & & & & & & & & & & & & & & & & & & & & 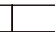 & & & & & & 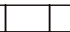 & 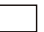 \\
\hline Guaramiranga & & & & $T$ & & & & & & & & & & & & & & & & & & & & & & & & L & & & & & & 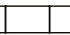 & \\
\hline Itapiúna & & & & I & T & & & & & & & T & & & & & & & & & & & & & & & & 1 & & & & & & L & 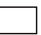 \\
\hline Maranguape & & & & T & T & & & & & & & T & & & & & & & & & & & & & & 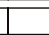 & 1 & 1 & & & & & & 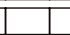 & \\
\hline Mulungu & & & & & & & & & & & & & & & & & & & & $T$ & & & & & & 1 & T & $T$ & & & & & D & & \\
\hline Pacoti & & & & 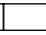 & & & & & & & & & & & & & & & & T & & & & & & 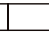 & T & $T$ & $\perp$ & & & 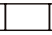 & 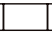 & I & \\
\hline Palmácia & & & & & & & & & & & & I & & & & & & & & I & & & 1 & & & 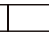 & 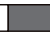 & L & 1 & & & L & L & 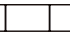 & \\
\hline Redenção & & & 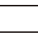 & & & 1 & & 1 & & & & & & & & & & & & & & & & & & & I & t & & & & & & 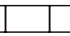 & \\
\hline Canindé & & & & $\mathrm{T}$ & T & & & & & & & & & & & & & & & & & & & & & & & 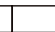 & & & & & & & \\
\hline Itatira & & & 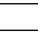 & 7 & & 7 & & 1 & & & 工 & T & & & & & & & & & & & & & & & & & & & & & & & \\
\hline Itapajé & & & & Eli & & 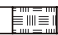 & & Ene & Eni= & & & 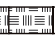 & & 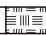 & & & & & & & & & 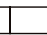 & & & 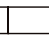 & 1 & $T$ & & I & d & ] & & & \\
\hline Itapipoca & & & & & & 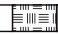 & & & & & Eili & & & 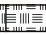 & & & & & & & & & & & & & & & & & & & & & \\
\hline Pedra Branca & & & & & & & & & & & 7 & T & & & & & & & & $T$ & & & T & & & T & & $T$ & & 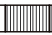 & 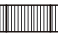 & 盂盂 & $\frac{\pi}{n t}$ & 盂盂 & \\
\hline Boa Viagem & & 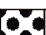 & & & & & & & & & & I & & & & & & & & 1 & & & 1 & & & t & & & & 60 & 60 & & & & \\
\hline Tamboril* & 82 & 0 & & & & 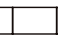 & & & & & 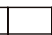 & I & & & & & & & & L & & & I & & & & & & & & & & & & \\
\hline Ceará & 148 & 146 & 2 & 16 & 5 & 11 & 2 & 4 & 22 & 69 & 31 & 175 & 3 & 33 & 10 & 3 & 6 & 0 & 0 & 0 & \begin{tabular}{|l|l}
4 \\
\end{tabular} & 0 & 0 & 0 & 0 & 0 & 7 & \begin{tabular}{|l|}
16 \\
\end{tabular} & 13 & 145 & 36 & 30 & 106 & \begin{tabular}{|l|l}
198 & 9 \\
\end{tabular} & 98 \\
\hline Óbitos & \begin{tabular}{|l|}
46 \\
\end{tabular} & \begin{tabular}{|l|}
45 \\
\end{tabular} & 0 & \begin{tabular}{|l|} 
\\
\end{tabular} & 0 & 2 & 0 & 1 & 6 & 11 & 5 & 29 & 2 & 6 & 2 & 0 & 3 & 0 & \begin{tabular}{|l|l|} 
& 0
\end{tabular} & 0 & 0 & $\begin{array}{ll}1 & 0\end{array}$ & 0 & 0 & 0 & 0 & 0 & 1 & 0 & 12 & 2 & 0 & \begin{tabular}{l|}
1 \\
\end{tabular} & \begin{tabular}{l|l}
4 &
\end{tabular} & 2 \\
\hline
\end{tabular}

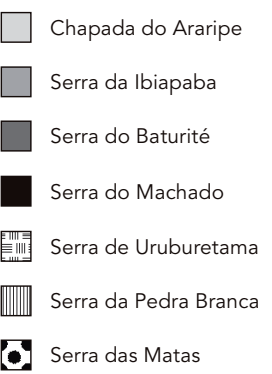


Figura 2 (continuação)

Distribuição dos casos humanos de peste pelas regiões pestígenas do Estado do Ceará, Brasil, 1935 a 2004.

\section{2b) $1970-2004$}

\begin{tabular}{|c|c|c|c|c|c|c|c|c|c|c|c|c|c|c|c|c|c|c|c|c|c|c|c|c|c|c|c|c|c|c|c|c|c|c|c|}
\hline Municipio & 70 & 71 & \begin{tabular}{|l|}
72 \\
\end{tabular} & 73 & 74 & 75 & 76 & 77 & 78 & 79 & 80 & 81 & 82 & 83 & 84 & 85 & 86 & 87 & \begin{tabular}{|l|}
88 \\
\end{tabular} & 89 & \begin{tabular}{|l|l|}
90 & -1 \\
\end{tabular} & \begin{tabular}{l|}
91 \\
\end{tabular} & 92 & \begin{tabular}{l|l}
93 & -1 \\
\end{tabular} & 94 & 95 & 96 & & \begin{tabular}{l|l}
98 & \\
\end{tabular} & 99 & \begin{tabular}{l|l}
00 & \\
\end{tabular} & \begin{tabular}{|l|}
01 \\
\end{tabular} & \begin{tabular}{|l|}
02 \\
\end{tabular} & 03 & 04 \\
\hline Abaiara & & & & & & & & & & & & & & & & & & & & & & & & & & & & & & & & & & & \\
\hline Araripe & & & & & & & & & & & & & & & & & & & & & & & & & & & & & & & & & & & \\
\hline Barbalha & & & & & & & & & & & & & & & & & & & & & & & & & & & & & & & & & & & \\
\hline Brejo Santo & & & & & & & & & & & & & & & & & & & & & & & & & & & & & & & & & & & \\
\hline Crato & & & & & & & & & & & 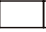 & & & & & 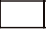 & T & T & & & & & & & & & & L & & & & & I & $\square$ & 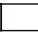 \\
\hline Jardim & & & & & & & I & & 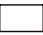 & I & 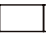 & & 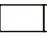 & & & $\square$ & I & L & 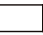 & L & & & & 1 & L & L & & L & & & & 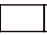 & 1 & 1 & 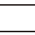 \\
\hline Missão Velha & & & & & & & T & & 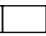 & I & 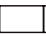 & & 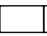 & & & + & L & L & + & 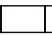 & & & & 1 & & & & L & & & & 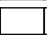 & 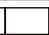 & 1 & L \\
\hline Nova Olinda & 7 & & & & & & & & 7 & 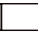 & 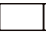 & & & & & 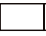 & & 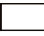 & & & & & & I & & & & & & & & 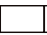 & D & L & 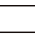 \\
\hline Porteiras & I & & & & & & & & 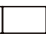 & & 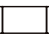 & & & & & $\square$ & L & E & & & & & & I & & & & & & & & 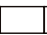 & 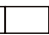 & 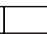 & 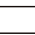 \\
\hline Santana do Cariri & 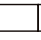 & & & & & & & & & & & & & & & 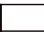 & & 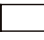 & & & & & & & & & & & & & & 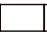 & 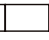 & L & 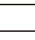 \\
\hline Guaraciaba do Norte & & & & & & & & & & & & & & & & & & 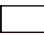 & & & & & & & & & & & & & & 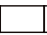 & 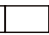 & 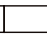 & 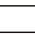 \\
\hline Ipu & & & & & & & & & & & & & & & & & & 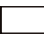 & & & & & & & & & & L & & & & 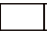 & 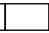 & L & 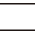 \\
\hline |pueiras & & & & & & & & & & & & & & & & & & & & & & & & & & & & & & & & 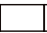 & . & 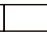 & \\
\hline Ibiapina & & & & & & & & & & & I & & & & & & 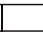 & 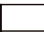 & & & & & & & & & & 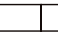 & & & 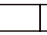 & 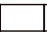 & L & G & \\
\hline Poranga & & & & & & & $T$ & & $\sigma_{0}$ & & L & & & & 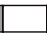 & 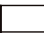 & 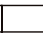 & $\mathrm{H}$ & 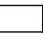 & & & & & I & 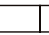 & 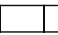 & & L & 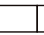 & 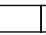 & 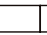 & E & I & L & \\
\hline São Benedito & & & & & & & & & & & & & & & & 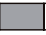 & I & L & & & & & & & & & & & & & - & $\mathrm{E}$ & 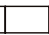 & 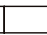 & \\
\hline \begin{tabular}{|l|l} 
Tianguá \\
\end{tabular} & & & & & & & T & & & I & & & & & & $\square$ & T & 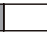 & $\square$ & & & & & & & L & & L & & & & 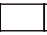 & 1 & 1 & 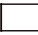 \\
\hline Ubajara & & & & & & & & & & & & & & & & 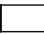 & T & 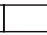 & & & & & & $\perp$ & & & & 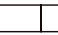 & & & & E & 1 & 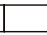 & - \\
\hline Viçosa do Ceará & $T$ & & & & & & & & & I & & & & & & 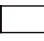 & I & 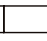 & & & & & & & & & & & & & & & & 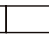 & \\
\hline Aratuba & & & & & & & & & 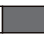 & & & & & & & & & & & & & & & & & & & & & & & E & & 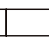 & \\
\hline Baturité & & & & & & & & & & & & & & & & 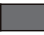 & $\mathrm{T}$ & I & & & & & & I & & & & 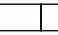 & & & & 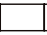 & 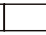 & L & 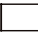 \\
\hline Capistrano & & & & & & & & & & & & & & & & & T & & & & & & & $\mathrm{T}$ & & & & 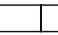 & & & & E & 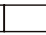 & 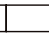 & 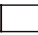 \\
\hline Guaramiranga & & & & & & & & & & & & & & & & - & & & & & & & & & & & & & & & & 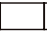 & & 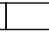 & 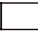 \\
\hline Itapiúna & & & & & & & & & & & & & & & & & & & & & & & & & & & & & & & & 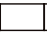 & . & 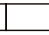 & \\
\hline Maranguape & & & & & & & & & & & & & & & & & & & & & & & & & & & & & & & & & & 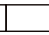 & \\
\hline Mulungu & & & & & & & & & & & & & & & & & & 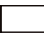 & & & & & & & & & & & & & & 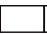 & 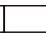 & D & \\
\hline Pacoti & & & & & & & & & & & & & & & & - & L & 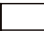 & & & & & & & & L & & L & & 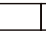 & 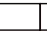 & L & I & 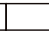 & 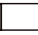 \\
\hline Palmácia & & & & & & & & & & & & & & & & 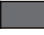 & I & L & & I & L & & & I & & L & L & L & & & 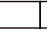 & E & I & L & 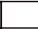 \\
\hline Redenção & & & & & & & & & & & & & & & & 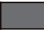 & & & & & & & & & & & & 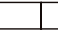 & & & & 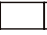 & 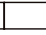 & 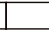 & \\
\hline Canindé & & & & & & & & & & & & & & & & & & & & & & & & & & & & & & & & & & & \\
\hline Itatira & & & & & & & & & & & & & & & & & & & & & & & & & & & & & & & & L & . & - & \\
\hline Itapajé & & & & & & & & & & & & & & & & & & & & & & & & & & & & & & & & 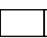 & & G & $\square$ \\
\hline \begin{tabular}{|l|} 
Itapipoca \\
\end{tabular} & & & & & & & & & & & & & & & & & & & & & & & & & & & & & & & & & & & \\
\hline Pedra Branca & 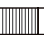 & & 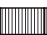 & 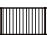 & & & & & & & & & & & & & & & & & & & & & & & & & & & & & & 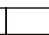 & \\
\hline Boa Viagem & 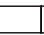 & & \begin{tabular}{|l|l|} 
& \\
\end{tabular} & $\square$ & & & & & 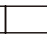 & & 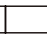 & & & & 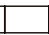 & 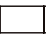 & $\mathrm{I}$ & 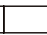 & 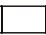 & & & & & 1 & & 口 & 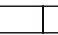 & 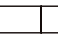 & & & & 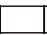 & 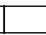 & 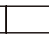 & 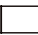 \\
\hline Tamboril* & & & & . & & T & & 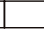 & 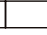 & I & 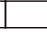 & & & & & 7 & I & I & & & & & & & & & & & & & & & 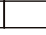 & 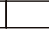 & 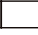 \\
\hline Ceará & 79 & 122 & 107 & 131 & 0 & 127 & 95 & 1 & 11 & 0 & 94 & 59 & 128 & 66 & 19 & 33 & \begin{tabular}{|l|}
4 \\
\end{tabular} & \begin{tabular}{|l|}
0 \\
\end{tabular} & 0 & 0 & 0 & 0 & 0 & 0 & \begin{tabular}{|l|}
1 \\
\end{tabular} & \begin{tabular}{|l|}
0 \\
\end{tabular} & \begin{tabular}{|l|}
1 \\
\end{tabular} & 1 & 0 & 0 & 0 & \begin{tabular}{|l|}
0 \\
\end{tabular} & 0 & 0 & 0 \\
\hline Óbitos & 0 & 6 & \begin{tabular}{|l|}
1 \\
\end{tabular} & 3 & 0 & 0 & 1 & 0 & 0 & 0 & 0 & \begin{tabular}{|l|}
0 \\
\end{tabular} & 0 & 0 & 0 & \begin{tabular}{|l|}
0 \\
\end{tabular} & 0 & 0 & 0 & 0 & 0 & \begin{tabular}{|l|}
0 \\
\end{tabular} & 0 & 0 & & \begin{tabular}{|l|}
0 \\
\end{tabular} & \begin{tabular}{|l|}
0 \\
\end{tabular} & \begin{tabular}{l|l}
0 \\
\end{tabular} & 0 & 0 & 0 & \begin{tabular}{|l|}
0 \\
\end{tabular} & 0 & 0 & 0 \\
\hline
\end{tabular}
$\square$ Chapada do Araripe
$\square$ Serra da lbiapaba
$\square$ Serra do Baturité
- Serra do Machado
Elli: Serra de Uruburetama
|III Serra da Pedra Branca
[-. Serra das Matas

\footnotetext{
* Monsenhor Tabosa.
} 
(Figura 1, Tabela 1). Os primeiros casos humanos foram notificados em 1938 e os últimos em 1948, em Itapipoca e Itapajé (Figura 2).

\section{Foco da Serra da Pedra Branca (CERES 8)}

O Complexo Ecológico da Serra da Pedra Branca é constituído por um único município localizado na Ceres 8 (Figura 1, Tabela 1). A ocorrência de casos humanos foi registrada a partir de 1964, também como uma extensão do foco da serra de Baturité, e o último caso humano ocorreu em 1974 (Figura 2).

\section{Foco da Serra das Matas (CERES 5 e 15)}

O Complexo Ecológico da Serra das Matas é constituído por dois municípios e as atividades de vigilância do foco estão sob a responsabilidade de duas CERES: a 5 (um município) e a 15 (um) (Figura 1, Tabela 1). Deve-se ressaltar que a jurisdição da CERES 5 estende-se ao foco da Serra do Machado e a da 15 ao da Serra da Ibiapaba. Em Boa Viagem houve notificações em 1936, 1964 (originado de Baturité) e 1965. Em maio de 1935 houve 27 casos em Monsenhor Tabosa, que na época era um povoado do Município de Tamboril.

\section{Discussão}

O comportamento epidemiológico da peste no Estado do Ceará entre 1935/2004 caracterizou-se pela ocorrência de diferentes padrões de circulação da enfermidade na população humana, alternando-se momentos de elevadas incidências nos principais focos conhecidos, seguidos por outros momentos de baixa incidência ou mesmo ausência completa de casos (Figura 2), o que possibilita estabelecer uma periodização cronológica com características epidemiológicas próprias. Por os focos cearenses se incluírem entre os principais do país, esta análise, que cobre um intervalo de aproximadamente 70 anos, assume um papel relevante em termos do conhecimento da tendência secular dessa zoonose e das lições para sua vigilância e controle no momento atual.

O primeiro período identificado, de 1935 a 1951 (17 anos), caracterizou-se pela ocorrência de elevadas incidências anuais, com uma média de 38 casos por ano, e substancial letalidade (foram notificados 683 casos e 162 óbitos, letalidade de $24 \%$ ) em decorrência da inexistência de recursos profiláticos e terapêuticos eficazes e também pela precariedade dos sistemas assistencial e de vigilância da época. Nas atividades de controle usaram-se as iscas raticidas à base do arsênico, o lança-chamas e o cianogás (cianeto de cálcio) a partir de 1941 e as iscas de 1080 (fluoroacetato de sódio) após 1950. Às vezes, contudo, o combate era feito com armadilhas toscas, como a arataca e o mundéu, e mesmo a pauladas. $\mathrm{O}$ combate às pulgas evoluiu da emulsão de sabão em querosene para os inseticidas de ação residual, o DDT e o BHC, a partir de 1948. O arsenal terapêutico disponível era o sublimado corrosivo (bicloreto de mercúrio 1:1.000), poções com o ácido fênico e os soro e vacina antipestosos, todos ineficazes. Os antimicrobianos comprovadamente eficazes tornaram-se disponíveis somente no final dessa fase $9,10,11$.

No segundo período, de 1952 a 1960 (nove anos), praticamente não houve atividade pestosa na maioria dos anos, com o registro de apenas quatro casos e nenhum óbito. Nesse momento, as investigações ainda eram incipientes pois tanto o SNP como o DNERu tinham como dogma que a peste se encerrava no binômio rato (Rattus rattus rattus, Linnaeus, 1758) e a sua pulga, a Xenopsylla cheopis (Rothschild, 1903) o que, aliado ao pragmatismo dos seus dirigentes, que visavam exclusivamente ao controle da zoonose, obtido com a despulização e os cuidados com o meio ambiente, dificultou a realização de pesquisas e conseqüentemente a definição dos reais caracteres epidemiológicos do agravo. O principal indicador da atividade pestosa, apesar de mais falho, era o caso humano e é sabido que nem todos chegavam ao conhecimento das autoridades sanitárias 12 .

É interessante salientar que nesse período já havia condições de se identificar a atividade pestosa, uma vez que com as técnicas bacteriológicas então disponíveis podia-se diagnosticar a infecção nos roedores 6,12. O grande entrave foi a atitude adotada pelas autoridades de saúde que não aceitavam a existência da peste silvestre, apesar das evidências de sua ocorrência 6,9,12, o que impossibilitou a realização de inquéritos. Tratase, portanto, de um silêncio relativo tendo em vista que se refere apenas a casos humanos, dado não existirem levantamentos sistemáticos sobre a circulação do bacilo na natureza. Essa circulação passou a ser detectada a partir de 1966, já no terceiro período, com o Plano Piloto de Peste em Exu (PPP), que empregando somente os exames bacteriológicos alcançou excelentes resultados tanto na peste humana quanto na identificação da atividade pestosa nos roedores reservatórios/ hospedeiros e seus ectoparasitos 13,14,15.

No terceiro período, de 1961 a 1986 (26 anos), houve novamente um aumento da incidência, com um total de 1.861 casos e 33 óbitos notificados (letalidade de $1,8 \%$ ), com uma média anual de 71 casos. Esse período distinguiu-se por 
apresentar elevadas incidências de casos e letalidade muito inferior ao primeiro período, pois já se dispunha de inseticidas e antimicrobianos eficazes e de sistemas de vigilância e assistência mais efetivos. Nesse período, na década de 80, merecem destaque a epidemia ocorrida na Serra de Baturité, de grande repercussão nacional 16, e a intensa atividade detectada na serra da Ibiapaba (Figura 2). Entretanto, os episódios de epidemização verdadeira, isto é, a ocorrência de mais de dez casos humanos na mesma habitação nos sítios ou fazendas e povoados, muito comuns no primeiro período (1935/1951), tornaram-se excepcionalmente raros.

O quarto período, de 1987 a 2004 (18 anos), apresentou baixíssima incidência de casos humanos, somente três, ao lado de evidências importantes da circulação do bacilo pestoso em reservatórios silvestres e domésticos, obtidas graças à agregação de novas estratégias e tecnologias à vigilância da zoonose, especialmente a vigilância sorológica, implantada no Brasil no início da década de 80 e no Ceará em 1987 17,18,19. A despeito da redução da ocorrência de peste humana nos anos recentes (Figura 2), a circulação do bacilo pestoso freqüentemente é detectada quando uma investigação rigorosa é realizada. Assim sendo, a vigilância sorológica detectou elevação do número de animais com anticorpos antipestosos a partir de 1995, com picos de ativação em 1997 e 2001 em todos os focos trabalhados, gerando o desencadeamento das ações de controle, com uma única notificação em 1997 20,21. O acervo de conhecimentos acumulados 2,15 e as tecnologias e estratégias de intervenção desenvolvidas e utilizadas 8 principalmente a partir da década de 70 , permitiram reduzir a morbimortalidade a níveis mínimos nesse período, contrastando com o que ocorria no primeiro.

Os focos são delimitados pela ocorrência de peste humana ou animal e caracteristicamente são limitados geograficamente, localizandose em terras elevadas e sujeitos a um regime de chuvas diferente do que predomina nas regiões circunvizinhas. Quando o clima é favorável e as colheitas são abundantes ocorrem as expansões, com os limites dos focos sendo ultrapassados graças ao aumento da densidade dos reservatórios, criando-se uma situação de risco para as zonas indenes 6,22.

$\mathrm{O}$ processo de municipalização levou à descentralização das ações de controle das ende- mias a partir de 1999, com as áreas pestígenas do estado passando a ser gerenciadas diretamente pelo poder municipal. Um aspecto relevante a se considerar no que diz respeito à organização da vigilância e controle da zoonose é o impacto da distribuição das áreas de um mesmo foco, que se constituem epidemiologicamente em unidades ou complexos ecológicos comuns, por diferentes unidades político-administrativas de gestão, as CERES (Figura 1, Tabela 1). Para efeito de gestão regionalizada das ações e serviços de saúde, a SESA/CE dividiu o estado em 21 células, sendo que os focos distribuem-se por 12 delas. A descentralização da gestão das ações de saúde, de uma maneira geral e do PCP em particular, fragmentou os focos por diferentes regionais, fracionando o planejamento, o gerenciamento e a execução das ações pertinentes a uma mesma área pestígena: o foco da Serra das Matas, por exemplo, é compartilhado por duas CERES, a 5 e a 15. Em contrapartida, uma mesma CERES pode comportar duas áreas pestígenas como a 5 , que inclui o foco da Serra das Matas e o da Serra do Machado, e a 15, abrangendo o da Serra das Matas e o da Ibiapaba (Figura 1, Tabela 1).

O fracionamento dos focos por diferentes células pode dificultar a estruturação da vigilância da peste de forma mais efetiva, pois ela pode perder a sua unidade operacional por conta de concepções técnico-administrativas distintas dos gestores regionais. Os focos pestosos extrapolam a linha tênue dos limites administrativos e os gestores possuem autonomia exclusivamente para gerenciar as ações de saúde pertinentes à sua CERES ou ao seu município, o que exige que a SESA/CE e o Conselho de Secretários Municipais de Saúde (COSEMS) desempenhem plenamente os seus papéis, integrando as atividades. Tal situação justifica uma discussão aprofundada sobre as atribuições e a importância do núcleo estadual de vigilância e controle da peste na articulação das ações desenvolvidas, no sentido de promover ações de vigilância e controle das áreas de foco na sua totalidade, ao invés de separadas por limites administrativos municipais ou regionais, inexistentes para os hospedeiros e vetores. As ações de vigilância e controle, portanto, devem ser desenvolvidas na perspectiva de foco e não de unidade administrativa, o que exige maturidade técnica, gerencial e política, respeitando-se os princípios e fortalecendo incondicionalmente o SUS. 


\section{Resumo}

Após a sua introdução no Ceará, Brasil, em 1900, a peste focalizou-se em sete complexos ecológicos: Chapada do Araripe e Serras da Ibiapaba, do Baturité, do Machado, das Matas, da Pedra Branca e de Uruburetama. As atividades de vigilância nesses focos no período de 1935/2004 foram desenvolvidas sucessivamente pelo Departamento Nacional de Saúde, Serviço Nacional de Peste, Departamento Nacional de Endemias Rurais, Superintendência de Campanhas de Saúde Pública, Fundação Nacional de Saúde e finalmente pela Secretaria de Vigilância em Saúde. A análise dos dados levantados sobre a ocorrência de casos humanos nesses 70 anos permite identificar diferentes padrões de circulação da enfermidade na população humana, caracterizados pela alternância de momentos de elevadas incidências e de silêncio, possibilitando estabelecer uma periodização cronológica com características epidemiológicas próprias. Conclui-se desses resultados que a peste ainda deve ser considerada uma ameaça potencial, o que justifica revitalizar as ações de vigilância epidemiológica, o que só ocorrerá com o fortalecimento de todas as instâncias do Sistema Único de Saúde.

Peste; Yersinia pestis; Vigilância Epidemiológica; Zoonoses

\section{Colaboradores}

A proposta do artigo foi de R. J. S. Pontes e A. I. Aragão. O levantamento de dados nos arquivos da Fundação Nacional de Saúde (FUNASA) e da Secretaria Estadual de Saúde (SESA/CE) foi realizado por A. I. Aragão. O. J. Nascimento consolidou os dados e confeccionou a tabela e as figuras. Todos os autores participaram da discussão dos resultados e da elaboração do texto.

\section{Referências}

1. Gage KL, Kosoy MY. Natural history of plague: perspectives from more than a century of research. Ann Rev Entomol 2004; 50:505-28.

2. Perry RD, Fetherston JD. Yersinia pestis - etiologic agent of plague. Clin Microbiol Rev 1997; 10:35-66.

3. World Health Organization. Human plague in 2002 and 2003. Wkly Epidemiol Rec 2004; 79:301-8.

4. Higgins R. Emerging or re-emerging bacterial zoonotic diseases: bartonellosis, leptospirosis, Lyme borreliosis, plague. Rev Sci Tech 2004; 23:569-81.

5. Inglesby TV, Dennis TD, Henderson DA, Bartlett JD, Ascher MS, Eitzen E, et al. Plague as a biological weapon: medical and public health management. JAMA 2000; 17:2281-90.

6. Baltazard M. Viagem de estudo ao Brasil para a organização de um projeto de pesquisas sobre a peste. Rev Bras Malariol Doenças Trop 1968; 20:335-66.

7. World Health Organization. Plague in the Americas. Washington DC: Pan American Health Organization/World Health Organization; 1965. (Scientific Publication, 115).

8. Fundação Nacional de Saúde. Guia de vigilância epidemiológica. v. II. 5a Ed. Brasília: Fundação Nacional de Saúde; 2002.
9. Freitas CA. Histórias da peste e de outras endemias. Rio de Janeiro: Programa de Educação Continuada, Escola Nacional de Saúde Pública; 1998.

10. Freitas CA. Notícia sobre a peste no Nordeste. Rev Bras Malariol Doenças Trop 1957; 9:123-33.

11. Freitas CA. Saúde no Brasil: nomes e fatos. Recife: Editora Bagaço; 1998.

12. De La Barrera JM. Relatório sobre a peste no Brasil. Washington DC: Organização Pan-Americana da Saúde; 1960.

13. Baltazard M. Situação atual do trabalho de pesquisa sobre a peste no Brasil. Rev Bras Malariol Doenças Trop 1968; 20:367-70.

14. Baltazard M. Pesquisas sobre a peste no Brasil. Rev Bras Malariol Doenças Trop 1968; 20:371-90.

15. Baltazard M. Recherches sur la peste au Brésil. 4éme rapport. Bull Soc Pathol Exot 2004; 97 Suppl: 93-117.

16. Almeida CR, Almeida AMP, Brasil DP, Leal MF, Lima JWO. Situação da peste no Brasil no ano de 1982. In: Anais do XIX Congresso da Sociedade Brasileira de Medicina Tropical. Rio de Janeiro: Sociedade Brasileira de Medicina Tropical; 1983. p. 149.

17. Almeida CR, Almeida AMP, Vieira JBF, Guida U, Butler T. Plague in Brazil during two years of bacteriological and serological surveillance. Bull World Health Organ 1981; 59:591-7. 
18. Almeida AMP, Brasil DP, Melo MEB, Leal NC, Almeida CR. Importância dos carnívoros domésticos (cães e gatos) na epidemiologia da peste nos focos do Nordeste do Brasil. Cad Saúde Pública 1988; 4:49-55.

19. Almeida AMP, Leal NC, Carvalho FG, Dantas Sobrinho J, Almeida CR. Plague surveillance in Brazil: 1983 - 1992. Rev Inst Med Trop São Paulo 1995; 37:511-6.

20. Aragão AI, Seoane ACM, Leal TCA, Leal NC. Vigilância da peste no Estado do Ceará: 1991-1999. Rev Soc Bras Med Trop 2002; 35:143-8.
21. Leal NC, Almeida AMP. Diagnosis of plague and identification of virulence markers in Yersinia pestis by multiplex-PCR. Rev Inst Med Trop São Paulo; 1999; 41:339-42.

22. Duplantier JC, Duchemin J-B, Chanteau S, Carniel E. From the recent lessons of the Malagasy foci towards a global understanding of the factors involved in plague reemergence. Vet Res 2005; 36:437-53.

Recebido em 19/Jan/2006

Versão final reapresentada em 19/Mai/2006 Aprovado em 26/Jul/2006 